\title{
Observation of Confined Current Ribbon in JET Plasmas
}

\author{
E. R. Solano, ${ }^{1}$ P. J. Lomas, ${ }^{2}$ B. Alper, ${ }^{2}$ G. S. Xu,${ }^{3}$ Y. Andrew, ${ }^{2}$ G. Arnoux,${ }^{2}$ A. Boboc, ${ }^{2}$ L. Barrera, ${ }^{1}$ P. Belo, ${ }^{4}$ \\ M. N. A. Beurskens, ${ }^{2}$ M. Brix, ${ }^{2}$ K. Crombe, ${ }^{5}$ E. de la Luna, ${ }^{1}$ S. Devaux, ${ }^{6}$ T. Eich, ${ }^{6}$ S. Gerasimov, ${ }^{2}$ C. Giroud, ${ }^{2}$ D. Harting, ${ }^{7}$ \\ D. Howell, ${ }^{2}$ A. Huber, ${ }^{7}$ G. Kocsis, ${ }^{8}$ A. Korotkov, ${ }^{2}$ A. Lopez-Fraguas, ${ }^{1}$ M. F. F. Nave, ${ }^{4}$ E. Rachlew, ${ }^{9}$ F. Rimini, ${ }^{10}$ \\ S. Saarelma, ${ }^{2}$ A. Sirinelli, ${ }^{2}$ S. D. Pinches, ${ }^{2}$ H. Thomsen, ${ }^{11}$ L. Zabeo, ${ }^{2}$ and D. Zarzoso ${ }^{12}$ \\ JET EFDA, Culham Science Centre, Abingdon, OX14 3DB, United Kingdom \\ ${ }^{1}$ Laboratorio Nacional de Fusión, Asociación EURATOM-CIEMAT, 28040, Madrid, Spain \\ ${ }^{2}$ Euratom/CCFE Association, Culham Science Centre, Abingdon, Oxon, OX14 3DB, United Kingdom \\ ${ }^{3}$ Institute of Plasma Physics, Chinese Academy of Sciences, Hefei 230031, China \\ ${ }^{4}$ Associação EURATOM/IST, Instituto de Plasmas e Fusão Nuclear, Avenida Rovisco Pais, 1049-001, Lisbon, Portugal \\ ${ }^{5}$ Department of Applied Physics, Ghent University, Rozier 44, 9000 Gent, Belgium \\ ${ }^{6}$ Max-Planck-Institut für Plasmaphysik, EURATOM-Assoziation, D-85748 Garching, Germany \\ ${ }^{7}$ Forschungszentrum Jülich GmbH, Institut für Plasmaphysik, EURATOM-Assoziation, TEC, D-52425 Jülich, Germany \\ ${ }^{8}$ KFKI, Association EURATOM, Post Office Box 49, H-1525, Budapest, Hungary \\ ${ }^{9}$ Association EURATOM-VR, Department of Physics, SCI, KTH, SE-10691 Stockholm, Sweden \\ ${ }^{10}$ EFDA Close Support Unit, Culham Science Centre, Culham, OX14 3DB, United Kingdom \\ ${ }^{11}$ Max-Planck-Institut für Plasmaphysik, EURATOM-Assoziation, D-17491 Greifswald, Germany \\ ${ }^{12}$ Ecole Polytechnique, F-91128, Palaiseau Cedex, France \\ (Received 23 November 2009; published 7 May 2010)
}

\begin{abstract}
We report the identification of a localized current structure inside the JET plasma. It is a field-aligned closed helical ribbon, carrying current in the same direction as the background current profile (cocurrent), rotating toroidally with the ion velocity (corotating). It appears to be located at a flat spot in the plasma pressure profile, at the top of the pedestal. The structure appears spontaneously in low density, high rotation plasmas, and can last up to $1.4 \mathrm{~s}$, a time comparable to a local resistive time. It considerably delays the appearance of the first edge localized mode.
\end{abstract}

DOI: 10.1103/PhysRevLett.104.185003

PACS numbers: 52.55.Tn, 47.32.cf

Birkeland first described field-aligned astrophysical plasma current filaments (Birkeland currents) in 1908 [1]. Walén and Alfvén discussed such objects in the 1940s and 1950s [2,3] and experimental confirmation was described in the 1960s [4]. More recently, it has been shown that confined (closed field line) field-aligned magnetic vortices are possible stationary solutions of the ideal MHD equations [5] when the density profile is flat. In a stationary plasma the relation $\vec{\nabla} \times \vec{B}=\mu_{0} \vec{j}$ connects a magnetic vortex to a localized current structure, which may itself move with the bulk plasma: this would be a rotating current filament.

Experimental observations of short-lived filamentary current structures in magnetically confined plasmas have been described recently $[6,7]$. Here we report on a longlived localized current structure, observed in magnetically confined plasmas at the JET tokamak. It is associated with MHD fluctuations known as outer modes [8-10] at JET. We now identify the outer mode (OM) as a confined longlived rotating current filament or ribbon. Further, the presence of this localized current structure substantially alters plasma behavior: a quasistationary state is established in which sudden energy bursts (edge localized modes, ELMs) are suppressed. Usually in the high confinement regime $(H$ mode) a region of high gradients of density and temperature is formed, known as the pedestal. Without the OM the pedestal is eroded periodically and violently by ELMs. ELMs can lead to unacceptably high heat flux in a fusion reactor.

A very characteristic signature of the outer mode is displayed in the fast Fourier transform of the Mirnov signals (magnetic probes measuring the time derivative of the poloidal field): its harmonic structure, shown in Fig. 1(g) (other plots in that figure will be described later). Typically the fundamental harmonic frequency of the OM is $f \sim 5-10 \mathrm{kHz}$ and its toroidal mode number is $n=1$. Every subsequent harmonic has $n$ increased by 1 . In the pulse 75411 depicted in Fig. 1 the fundamental frequency is $f \sim 6 \mathrm{kHz}$ and harmonics are seen up to $\sim 42 \mathrm{kHz}$ ( $n=$ 7 ), but the mode is sometimes clearly recognizable up to $90 \mathrm{kHz}(n=15)$. This rich harmonic structure hints at strong localization of the current source that produces the magnetic fluctuation, which is observed in all Mirnov probes around the plasma. Plotted in Fig. 2 are signals from a toroidal array of Mirnov coils: discrete blips can be clearly seen, propagating toroidally with $f=6 \mathrm{kHz}$. The current structure that produces such blips rotates in the same direction as the ions in the plasma and carries excess current relative to the axisymmetric equilibrium current profile. Since the current feature is long-lived $(>1 \mathrm{~s})$, it must be located inside the magnetic separatrix. Since $n=$ 1 and the fundamental frequency is narrow, it must follow 


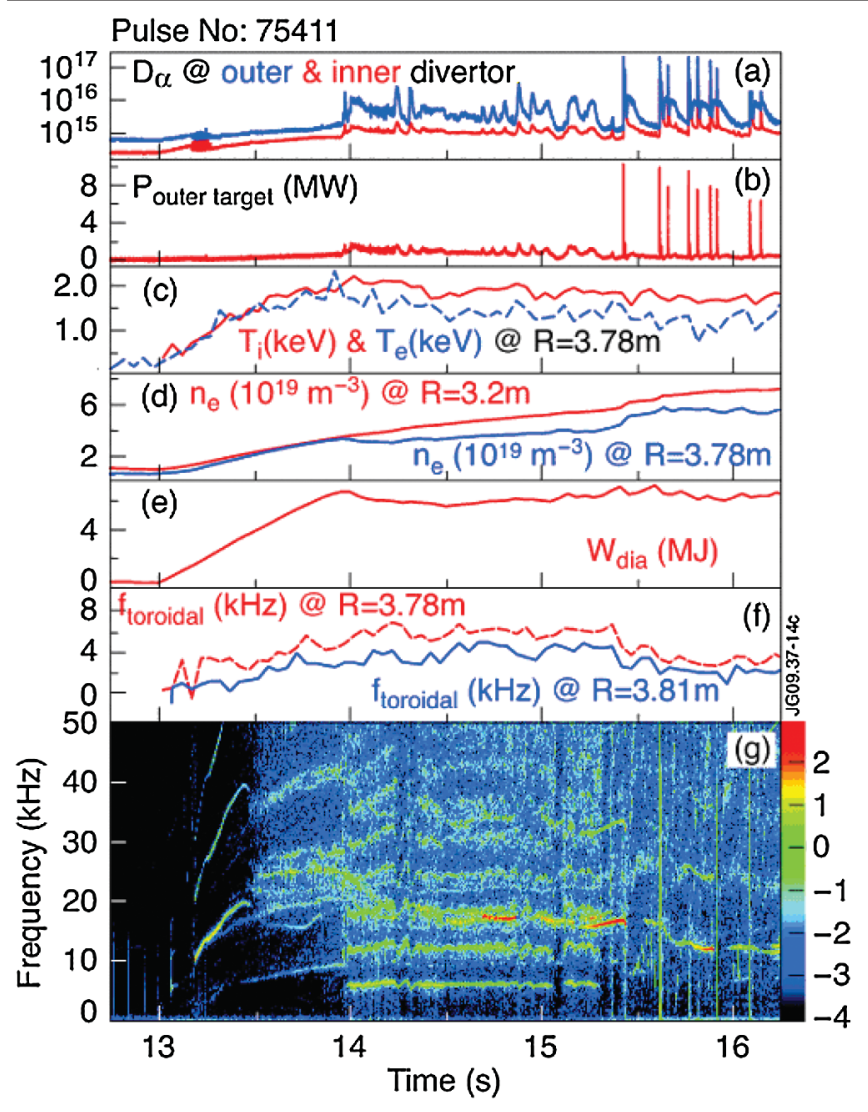

FIG. 1 (color). Time traces showing a long-lived outer mode, starting from $14 \mathrm{~s}$ to $15.38 \mathrm{~s}$, and its effect on plasma behavior: (a) rise in $D \alpha$; (b) rise in power to outer target; (c) drop in pedestal temperatures; (d) slower pedestal density rise, rising core density; (e) stationary energy; (f) toroidal rotation frequency at top and middle of pedestal; (g) fast Fourier transform of magnetic signal.

closed field lines, aligned with the magnetic field at a rational surface. The safety factor $q=m / n$ is used to describe the topology of closed field lines, where $m$ and $n$ are the poloidal and toroidal mode number, respectively. Plotted in Fig. 3 is a comparison of measured magnetic signals at various locations around the tokamak with simulated signals produced by rotation of a field-aligned current ribbon located at the $q=4,3$, or 2 surfaces. The current in the ribbon necessary to reproduce the measured signal height is quoted in each box. The scatter in simulated current values is representative of uncertainties in reconstruction of the plasma flux surfaces. Comparison of the shapes and phases of measured and simulated signals shows that the current structure is best simulated as a current ribbon with excess current of 100-300 A (plasma current is $2.5 \mathrm{MA})$, with $n=1, m=4(q=4)$, and toroidal width of the order $5 \%-10 \%$ of the toroidal circumference of the plasma, rotating toroidally at $6 \mathrm{kHz}$. The width of the ribbon is determined by the fastest observed change in $d B / d t$. Detailed equilibrium reconstruction is too sensitive to measurement and modeling inaccuracies, but compatible with $q=4$ being located at the flattop of

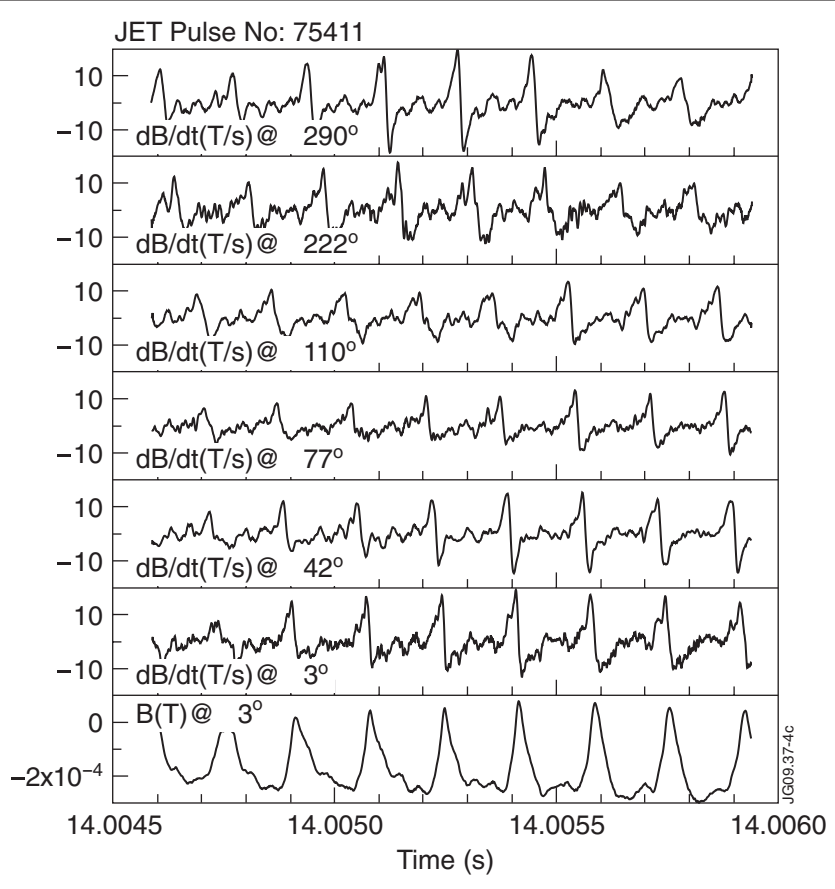

FIG. 2. Mirnov coil signals from toroidal array (outboard), showing toroidal propagation of magnetic feature in cocurrent direction. The lowest plot is the integrated signal from the coil at $3^{\circ}$.

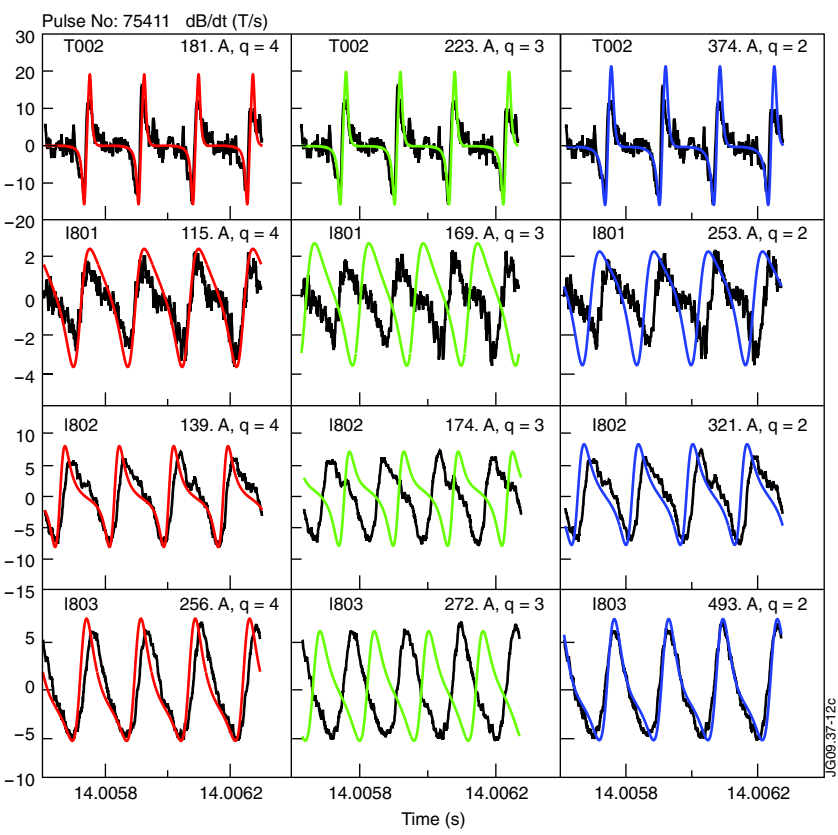

FIG. 3 (color online). Comparison of simulated signals (colored/grey) with data (black) in Mirnov coils located at the plasma outboard (top row), and three inboard coils located above, near, and below the plasma midplane, respectively. Simulated signals are generated with rotating current ribbons located at $q=4$ (red), $q=3$ (green), and $q=2$ (blue) flux surfaces. The currents quoted in each case are adjusted to match calibrated signal amplitude. 


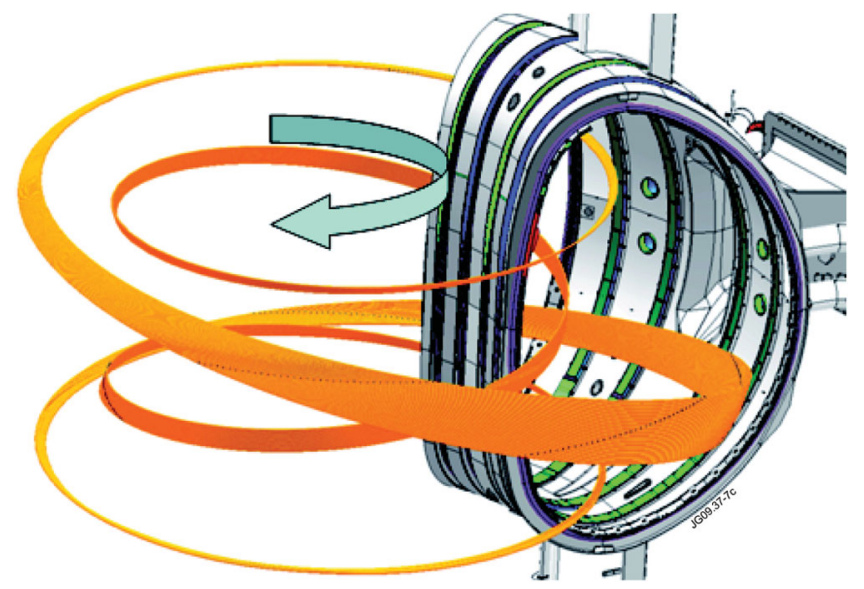

FIG. 4 (color). $q=4$ spinning current ribbon that provides best fit to magnetic signals from Mirnov coil set, with 100300 A.

the pressure pedestal. A representation of the $q=4$ current ribbon that best matches the data is shown in Fig. 4.

To identify the current source radial location we study the plasma profiles shown in Fig. 5. We assume that the fluid velocity is equal to the ion velocity, and that the current structure rotates with the plasma. From the charge-exchange measurements of the toroidal ion rotation frequency, we identify the radial position of the current structure as the location where toroidal rotation and mode frequencies match. From the start of the OM (Fig. 5, top three plots) it appears that the match occurs at the flattop of the density pedestal, just inboard of the maximum pressure

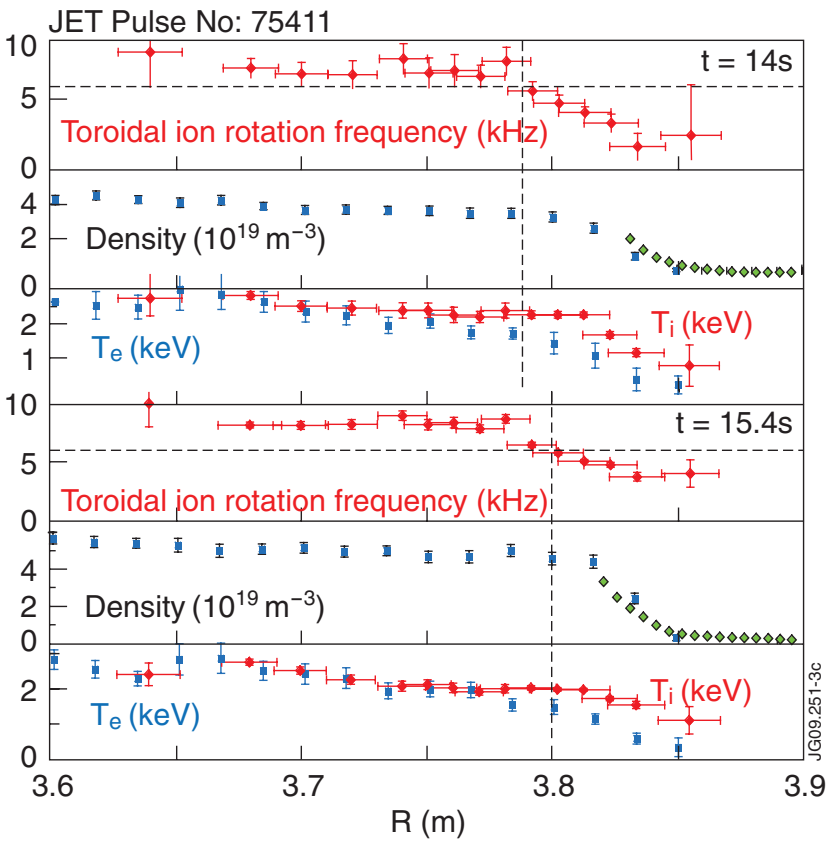

FIG. 5 (color). Profiles of toroidal rotation frequency, $n_{e}, T_{e}$, $T_{i}$ at start (top three plots) and end (lower three plots) of OM. Vertical line mark position where mode and plasma rotation frequencies are equal. gradient, at the edge of the ion rotation gradient region. Since the rotational shear is high around $6 \mathrm{kHz}$, the current structure must be well localized in the radial direction, hence the ribbon description. At the end of the OM phase (Fig. 5, lower plots) the toroidal rotation shear is somewhat eroded and the mode location shifts outwards by $2 \mathrm{~cm}$, still at the pressure pedestal flattop. After the first ELM the pedestal rotation frequency drops below $4 \mathrm{kHz}$, density rises, and the OM does not return.

Having thus established that the localized current structure is a $q=4$ field-aligned ribbon located at a profile position with no significant pressure gradient, but with some velocity shear, we turn to fluctuation measurements for clues on the consequent magnetic structure. Fluctuations of electron cyclotron emission in the $T_{e}$ gradient region, $X$-mode reflectometry, and edge channels of soft $\mathrm{x}$ rays all show at least 3 harmonics of the OM. All these fluctuations' measurements are sensitive to flux surface deformations and magnetic fields introduced by current structures. The largest fluctuations are seen in the gradient region of the pedestal, where diagnostic sensitivity is enhanced by local gradients. The fluctuation diagnostics cannot provide independent confirmation of the location of the current ribbon, but are compatible with its location at the flattop, where sensitivity is minimal. Electron cyclotron emission measurements show that all channels rise and fall together (in phase) as the field perturbation rotates past the antenna, confirming earlier results [9] that established the $\mathrm{OM}$ is not due to a magnetic island (tearing mode), to within the $1-2 \mathrm{~cm}$ spatial resolution of the diagnostic.

Operationally, OMs are often (but not exclusively) observed before the first ELM during, or immediately after, a hot-ion $H$-mode phase [11], obtained by reducing particle fueling (from external sources and wall) and operating at initially low density. In the experiments described here the plasma had high triangularity $(\delta=0.4)$, the current and toroidal field were, respectively, $2.5 \mathrm{MA}$ and $2.7 \mathrm{~T}$, and heating and rotation were provided by $15 \mathrm{MW}$ of coinjected neutral beam injection. The hot-ion $H$-mode regime was being used as a means to achieve ITER-relevant pedestal temperatures: up to $T_{e \text {, ped }}=2.8 \mathrm{keV}$ can be reached before the first ELM, if appropriate gas fueling is used to prolong the hot-ion regime, avoiding OMs and other MHD instabilities.

Time traces of an especially long OM were shown in Fig. 1. After the transition to $H$ mode, marked by the initial $D_{\alpha}$ drop at $t=13.2 \mathrm{~s}$, the pedestal $\left(n_{e}, T_{e}, T_{i}\right.$ at $R=$ $3.78 \mathrm{~m}$ ) rises as usual in an $H$ mode, until the OM begins at the time $t=13.98 \mathrm{~s}$. Then $D_{\alpha}$ rises, $T_{e \text {,ped }}$ begins to drop, while the pedestal toroidal rotation frequency (time resolution is $50 \mathrm{~ms}$ ) remains fairly constant. Density continues to rise, albeit slower than before. The energy confinement during the $\mathrm{OM}$ phase is similar to that of typical ELMy $H$ modes. Impurity content during the OM is characterized by $Z_{\text {eff }}$ of order $1.5-2$, normal compared to an 


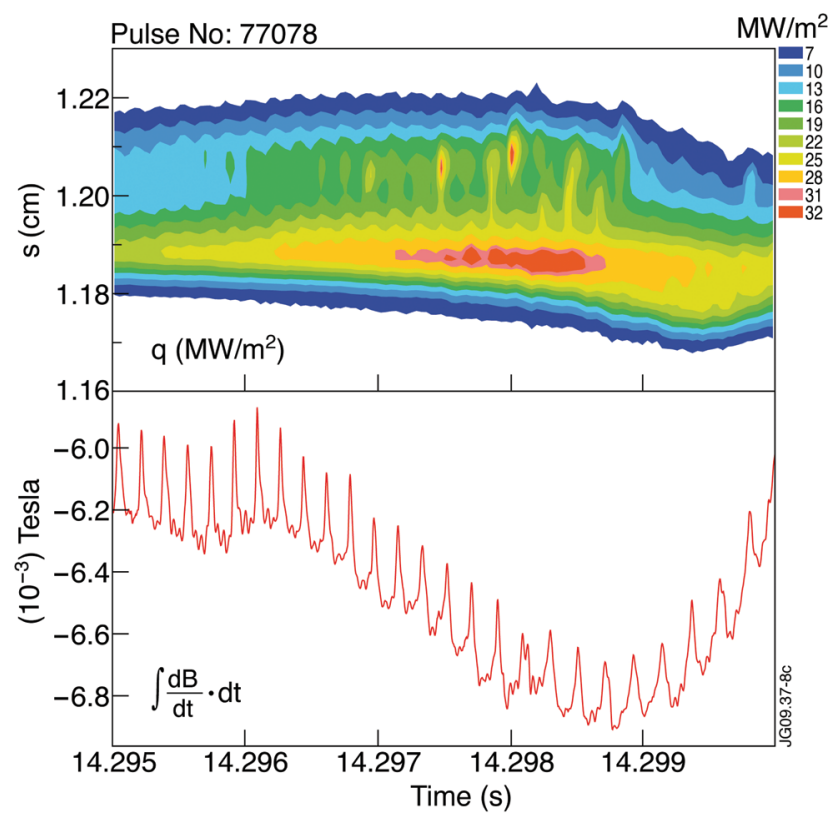

FIG. 6 (color). Heat flux contours at outer strike, measured with IR, and integrated magnetic signal showing correlation of heat pulses with rotation of current structure.

equivalent density ELMy $H$ mode. The OM survives 2 sawtooth crashes (at 14.23 and $14.87 \mathrm{~s}$ ), indicating that it is a robust feature of the plasma. There is a brief quiet interval between the end of the $\mathrm{OM}$ at $15.375 \mathrm{~s}$ and the ELM at $15.430 \mathrm{~s}$. The ELM-free period is prolonged by the presence of the OM [12]; a similar pulse with additional fueling $\left(1.9 \times 10^{21}\right.$ electron $/ \mathrm{s}$ instead of $\left.1.5 \times 10^{21} \mathrm{e} / \mathrm{s}\right)$ had no long-lived OMs and the first ELM occurred $1 \mathrm{~s}$ sooner, at $14.4 \mathrm{~s}$.

Clues on magnetic structure are provided by plasma footprint observations. The power outflux to the outer divertor target is measured with a fast infrared (IR) camera, viewing a narrow toroidal strip across the outer target. In pulse 75411 the time averaged heat flux during the $\mathrm{OM}$ is very similar in peak value and radial distribution to the ELM-averaged heat flux of the ELMy phase, taking into consideration that after ELMs there are large strike point position shifts [13]. But the frame rate was too slow to detect the $6 \mathrm{kHz}$ bursts. For pulse 77078, with an OM from 14.28 to $14.355 \mathrm{~s}$, the camera frame rate was especially set to $1 / 38 \mu \mathrm{s}(26 \mathrm{kHz})$, with an integration time of $27 \mu \mathrm{s}$, viewing only the outboard target. Soon after the mode onset the total power to the target increases, consistently with a loss of core confinement at that time. More interestingly, periodic bursts of heat arrive away from the maximum deposition location, as shown in Fig. 6, attributable to a toroidally localized "hose pipe." The observed heat deposition is consistent with the effect of a rotating current structure at the top of the pedestal: it can break toroidal symmetry and produce partial ergodisation of field lines increasing overall particle and heat flux across the pedestal. Additionally, at a specific toroidal location in the gradient region (away from the current structure) a flux tube can escape through the broken separatrix (a homoclinic tangle, as described in [14]) and lead to the toroidally localized heat pulses shown in Fig. 6.

From the theory point of view we should note that MHD studies of the plasma edge usually search for instabilities located in the gradient region. Such models do not predict formation of localized current structures in a low gradient region. A linear stability analysis before the onset of the OM shows that the plasma is quite far from the $n=1$ boundary. Whether the nonlinear evolution of either tearing or kink modes might lead to a saturated mode comparable to the OM is yet unknown. From the experimental evidence described above we speculate that the high $T_{e \text {,ped }}$ (ideal MHD) and/or the high rotation shear present in the plasma before the mode onset lead(s) to the circumstances necessary for localized current structure formation (closed MHD vortices), as implied in [5]. Another possibility is the bifurcation of the equilibrium field to a combined field produced by the background plasma plus a rotating helical perturbation, as sketched in [15]. Further work is needed to test these and other first-principles-based theoretical predictions.

Long-lived OMs in JET are very reminiscent of the quiescent $H$ mode ( $Q H$ mode) [16-18]. The current ribbon identified as the OM in JET may be related to the edge harmonic oscillation observed in $Q H$ modes of DIII-D and AUG. Like the OM, the edge harmonic oscillation produces a blip in the magnetic diagnostics and in the channels of the fluctuation diagnostics located in the pedestal gradient region, and replaces ELMs. So far there are no reports on the radial location of the current source that produces the edge harmonic oscillation fluctuations. Like the $Q H$ mode, a quasisteady $H$ mode as induced by the OM in JET might be a potentially useful fusion operating regime, provided it can be controlled in a predictable manner.

In summary, a spontaneously formed closed current ribbon has been observed in the JET tokamak. It is located at the pedestal top, it is long-lived, and it regulates transport across the plasma pedestal, significantly delaying the appearance of ELMs. Theory-based predictions of localized structures in ideal MHD can be developed to guide further research.

We are grateful to Tom Osborne, W. Suttrop, D. Borba, and D. M. McDonald for useful discussions. This work, supported by the European Communities under the contract of Association between EURATOM and the Laboratorio Nacional de Fusión, CIEMAT, was carried out within the framework of the European Fusion Development Agreement. This work was done under the JET-EFDA workprogramme [19].

[1] Kristian Birkeland, The Norwegian Aurora Polaris Expedition 1902-1903 (Aschehoug, Oslo, Norway, 1908). 
[2] C. Walén, Arkiv. f. Mat. Astr. o. Fys. Bd 30 A, No. 15, (1944); Bd 31 B, No. 3 (1944).

[3] H. Alfvén, Cosmical Electrodynamics (Clarendon Press, Oxford, England, 1953).

[4] W. D. Cummings and J. Dessler, J. Geophys. Res. 72, 1007 (1967).

[5] V. I. Petviashvili, Plasma Phys. Rep. 19, 256 (1993).

[6] G. Y. Antar et al., Nucl. Fusion 49, 032001 (2009).

[7] E. Martines et al., Plasma Phys. Controlled Fusion 51, 124053 (2009).

[8] M. F. F. Nave et al., Nucl. Fusion 35, 409 (1995).

[9] G. T. A. Huysmans et al., Nucl. Fusion 38, 179 (1998).

[10] C. P. Perez et al., Nucl. Fusion 44, 609 (2004).

[11] JET Team, presented by P. J. Lomas, in Plasma Physics and Controlled Nuclear Fusion Research 1994 (Proc. 15th Int. Conf. Seville, 1994) (IAEA, Vienna, 1995), Vol. I, p. 211.

[12] M.F. F. Nave et al., Nucl. Fusion 39, 1567 (1999).

[13] E. R. Solano et al., Nucl. Fusion 48, 065005 (2008).

[14] T. E. Evans et al., J. Phys. Conf. Ser. 7, 174 (2005).

[15] F.E. M. da Silveira and R. M. O. Galvão, Plasma Phys. Controlled Fusion 49, L11 (2007).

[16] K. H. Burrell et al., Phys. Plasmas 12, 056121 (2005).

[17] K.H. Burrell et al., Phys. Rev. Lett. 102, 155003 (2009).

[18] W. Suttrop et al., Nucl. Fusion 45, 721 (2005).

[19] See F. Romanelli and R. Kamendje, Nucl. Fusion 49, 104006 (2009). (All members of the JET-EFDA Collaboration appear in the appendix of this paper.) 